Supplementary Information

\title{
3-D Spiraling Self-Trapped Light Beams in Photochemical Systems
}

Derek R. Morim, Damian Bevern, Ignacio Vargas-Baca and Kalaichelvi Saravanamuttu*

Correspondence to:

Dr. Kalaichelvi Saravanamuttu

Associate Professor

Dept, of Chemistry and Chemical Biology,

McMaster University,

1280 Main St. West, Hamilton, Ontario L8S 4M1,

CANADA

Tel.: 19055258140 ext. 24615

Email: kalai@mcmaster.ca 


\section{S1 Preparation of photoresponsive gels}

\section{Materials}

3-(Trimethoxysilyl) propyl methacrylate (MAPTMS), $0.1 \mathrm{~N}$ hydrochloric acid, Pluronic ${ }^{\circledR} \mathrm{F}$ 127 polymer, and sodium iodide were purchased from Millipore Sigma. (Bis ( $\eta 5-$ cyclopentadien-1-yl)-bis(2, 6-difluoro-3-(1H-pyrrol-1-yl)-phenyl) titanium(IV) was obtained from Ciba Specialty Chemicals Inc., Canada. Tris(bipyridine)ruthenium(II) chloride hexahydrate was obtained from Strem Chemicals Inc.

\section{Photopolymerizable gel}

Adapted from our previous work ${ }^{1}$, this photopolymer was prepared by combining $1.1 \mathrm{~g}(5.5 \mathrm{x}$ $10^{-5}$ mol.) of $0.05 \mathrm{~N}$ hydrochloric acid (diluted from a standardized $0.1 \mathrm{~N} \mathrm{HCl}$ aqueous solution) and $17.6 \mathrm{~g}(0.0708 \mathrm{~mol})$ of 3-methacryloxypropyltrimethoxysilane. Approximately 5 minutes of stirring creates a homogeneous mixture, to which 0.05 wt. \% of (bis( $\eta 5-$ cyclopentadien-1-yl)-bis(2,6-difluoro-3-(1H-pyrrol-1-yl)-phenyl) titanium(IV) was added. The sample was shielded from ambient light with aluminum foil and stirred continuously for 6 days. $1.0 \mathrm{ml}$ of photosensitized sol was injected through a small opening into a $6 \mathrm{~mm}$-long cylindrical cell with flat and transparent windows. This organosiloxane sol was then uniformly irradiated in a homemade irradiation chamber with a reflective, aluminum foil interior to uniformly distribute the light onto the sample. White light from a quartz- tungstenhalogen lamp ( $3 \mathrm{~mW}$ ) was directed into the chamber for 8 minutes (Cole-Parmer 09790series, IL, USA), partially polymerizing the liquid into the gel that was employed for optical experiments.

\section{Photo-oxidation system}

Adapted from our previous work ${ }^{2}$, a solution of $\mathrm{Ru}(\mathrm{bpy})_{3} \mathrm{Cl}_{2}(1.3 \mathrm{mM})$ and $\mathrm{NaI}(0.2 \mathrm{M})$ was prepared with deionized $\mathrm{H}_{2} \mathrm{O}$ (Milli-Q ${ }^{\circledR}, 18.2 \mathrm{M} \Omega \mathrm{cm}$ ). $3 \mathrm{~mL}$ of this solution was added $1.0 \mathrm{~g}$ of Pluronic ${ }^{\circledR}$ F-127 and cooled below its lower critical solution temperature (LCST) using an ice bath and mixed with a glass rod until homogeneous. The sample was then injected into the opening of a 6 $\mathrm{mm}$ ring cell while coldand left at room temperature (shielded with aluminium foil) until a gel formed. 


$$
\begin{aligned}
& {\left[\mathrm{Ru}(\mathrm{bpy})_{3}\right]^{2+}+\mathrm{hv} \longrightarrow\left[\mathrm{Ru}(\mathrm{bpy})_{3}\right]^{2+*}} \\
& {\left[\mathrm{Ru}(\mathrm{bpy})_{3}\right]^{2+*}+\mathrm{I}^{-} \longrightarrow\left[\mathrm{Ru}(\mathrm{bpy})_{3}\right]^{+}+\mathrm{I}^{\bullet}} \\
& \mathrm{I}^{\cdot}+\mathrm{I}^{-} \longrightarrow \mathrm{I}_{2} \cdot \\
& \mathrm{I}_{2}^{\cdot-}+\mathrm{I}_{2}^{--} \longrightarrow \mathrm{I}^{-}+\mathrm{I}^{-} \\
& {\left[\mathrm{Ru}(\mathrm{bpy})_{3}\right]^{+}+\mathrm{I}_{2}{ }^{--} \longrightarrow\left[\mathrm{Ru}(\mathrm{bpy})_{3}\right]^{2+}+2 \mathrm{I}^{-}}
\end{aligned}
$$

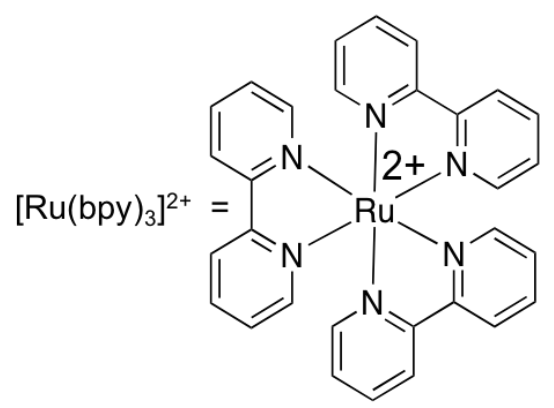

Scheme S1 Photochemical conversion of iodide to triiodide occurs through multiple radical processes.

\section{S2 Optical assembly}

Optical experiments were carried on an assembly adapted from our previous studies (Figure S1). ${ }^{1}$ The excitation source was the $\mathrm{TEM}_{00}$ mode (Gaussian beam, M2<1.1) of c,w. visible (532 nm) light emitted by a diode-pumped solid-state laser (Verdi V2, Coherent, Inc., California, USA). The output beam was passed through a $\lambda / 2$ waveplate and split into two beams by a polarizing beam splitter cube, creating two orthogonally polarized beams. The $\lambda / 2$ waveplate orientation was adjusted to ensure that the intensity of each beam was equal. The beams were directed by mirrors and a second beam splitter cube, through a set of filters and a planoconvex lens [focal length $=75.6 \mathrm{~mm}$ ], which focused each of the beams to a diameter of $20 \mu \mathrm{m}$ onto the entrance window of the sample cell. The separation distance $(\Delta s)$ and separation angle $(\theta)$ of the beams was tuned by varying the orientation of the beam splitter cube.

The spatial intensity profile of the beams at the exit face of the cell was imaged by a pair of planoconvex lenses (f. $1 .=75.6 \mathrm{~mm}$ and $\mathrm{f} .1 .=250.0 \mathrm{~mm}$ ) onto a charge-coupled device $(\mathrm{CCD})$ camera [2040 horizontal $\times 1532$ vertical pixels; pixel size $=3.20 \mu \mathrm{m} \times 3.20 \mu \mathrm{m}$; WinCamD XHR, DataRay Inc., California, USA]. Neutral density filters (F) (VARM, Coherent, Inc.) were used to prevent saturation of the CCD camera. All components are translatable along the $z$ direction of the optical rail with a resolution of $0.25 \mathrm{~mm}$. Images were collected every second for 50 second data logs on DataRay software (Version 7.1H25Bk), which calculates beam diameter $\left(1 / \mathrm{e}^{2}\right)$ and relative peak intensity. Corrections for image magnification by the lenses $(\times 3.3)$ and filters were applied to the images and normalized for better visualization of the beam (the diffracted beam is less intense than the self-trapped beam). The effective beam diameter corresponds to the circular area of all pixels with relative intensity $>13.5 \%\left(1 / \mathrm{e}^{2}\right)$ of the maximum (peak) intensity. 


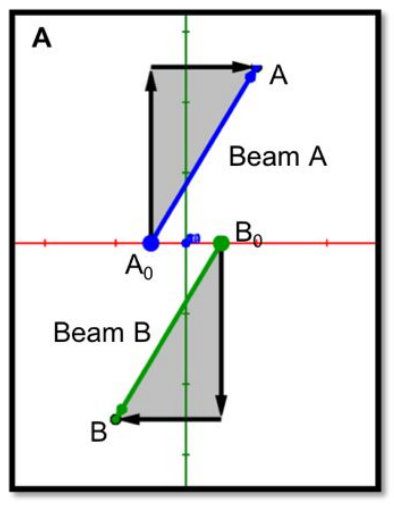

CCD L3
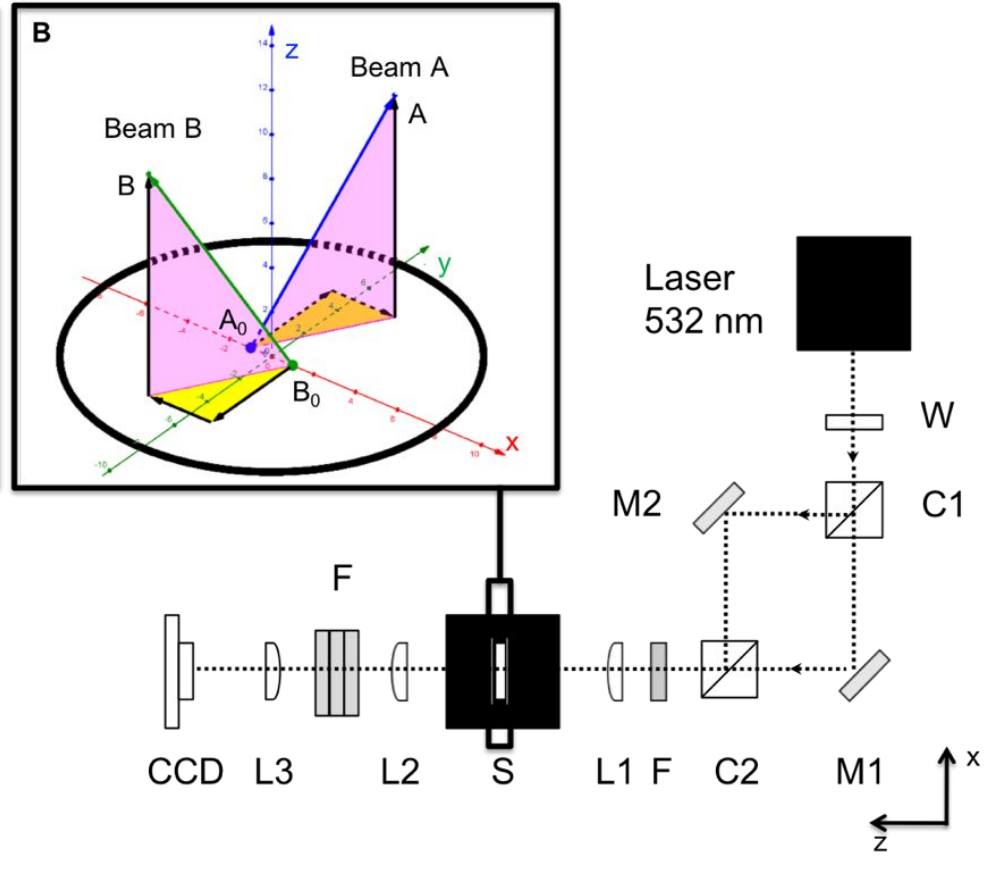

L

Figure S1 Optical assembly employed to launch two skewed laser beams into photoresponsive gels. $\mathrm{W}=\lambda / 2$ waveplate; $\mathrm{C} 1, \mathrm{C} 2=$ polarizing beamsplitter cubes; $\mathrm{L} 1, \mathrm{~L} 2$, $\mathrm{L} 3$ = planoconvex lenses; $\mathrm{F}=$ filters, $\mathrm{S}=$ sample, and $\mathrm{CCD}=$ charge coupled device camera for imaging the optical profile of the output of the sample. (A) The beam vectors in the $x y$ plane. (B) The beam vectors in 3D representing the trajectory of the beams under linear conditions.

\section{S3 Calculations of beam trajectories}

The trajectories on the beams were determined from the positions of their centres at the entrance: $\mathbf{p}_{\mathbf{0}}$ $=\left(\boldsymbol{x}_{\mathbf{0}}, \boldsymbol{y}_{\mathbf{0}}, \mathbf{z}_{\mathbf{0}}\right)$, and exit: $\mathbf{p}_{\boldsymbol{f}}=\left(\boldsymbol{x}_{\boldsymbol{f}}, \boldsymbol{y}_{\boldsymbol{f}}, \mathbf{z}_{\boldsymbol{f}}\right)$ faces of the sample under linear conditions. From those values, the beam propagation vectors were obtained:

$\vec{v}=\left(x_{f}-x_{0}, y_{f}-y_{0}, z_{f}-z_{f}\right)$

The separation distance between the beams $\overrightarrow{\boldsymbol{a}}$ and $\overrightarrow{\boldsymbol{b}}$ at the input of the sample ( $\Delta \mathrm{s})$ can be determined by: $\Delta s=x_{a 0}-x_{b 0} \quad[\mathrm{~S} 2]$

and

$\Delta s=\sqrt{\left(x_{a 0}-x_{b 0}\right)^{2}+\left(y_{a 0}-y_{b 0}\right)^{2}}$

in case of any small deviation in the $y$ position of the beams. The angular separation between the beams, $\theta$, was determined through:

$\vec{a} \cdot \vec{b}=\overrightarrow{\|a\|} \overrightarrow{\|b\|} \cos \theta$

where the magnitude of the vectors is determined according to:

$$
\overrightarrow{\|v\|}=\sqrt{\left(x_{0}-x_{f}\right)^{2}+\left(y_{0}-y_{f}\right)^{2}+\left(z_{0}-z_{f}\right)^{2}}
$$

Finally, the minimum separation of the beams in space $\left(\Delta \mathrm{s}_{\min }\right)$ can be determined by finding a vector perpendicular to both beams such that: $\overrightarrow{\boldsymbol{a}} \cdot \overrightarrow{\boldsymbol{c}}=\mathbf{0}=\overrightarrow{\boldsymbol{b}} \cdot \overrightarrow{\boldsymbol{c}}$. 
As $\vec{a}$ and $\vec{b}$ are known, the result is a system of equations that can be solved to determine $\vec{c}$. Since the $\mathrm{x}$ and y components of vectors $\vec{a}$ and $\vec{b}$ tend to be small compared to the propagation component $\mathrm{z}$, it is possible to keep $\Delta s_{\min }$ constant by ensuring that the ratio of the $\mathrm{x}$ and $\mathrm{y}$ components remains approximately the same.

We define the rotation angle as the angular difference in the orientation of the beam pair at the exit face under linear conditions and nonlinear conditions, i.e. prior to and after laser-induced photochemical reactions in the medium. The twist angle corresponds to the angular difference in the orientation of the beam pair at the entrance face and at the exit face under nonlinear conditions (Figure S2).
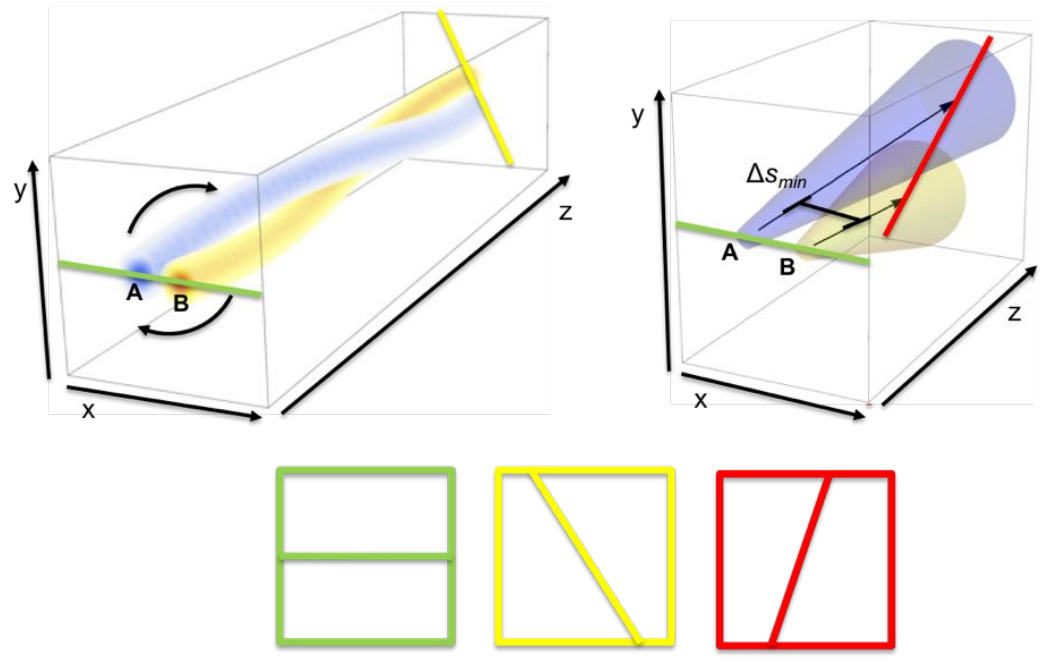

Entrance

Exit (nonlinear)

Exit (linear)

Rotation angle $=$ exit (nonlinear) - exit (linear)

Twist angle $=$ exit (nonlinear) - Entrance
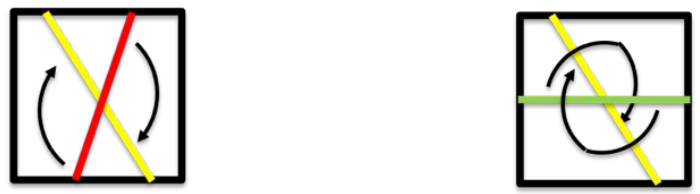

Figure S2 Definitions of rotation angle and twist angle. 


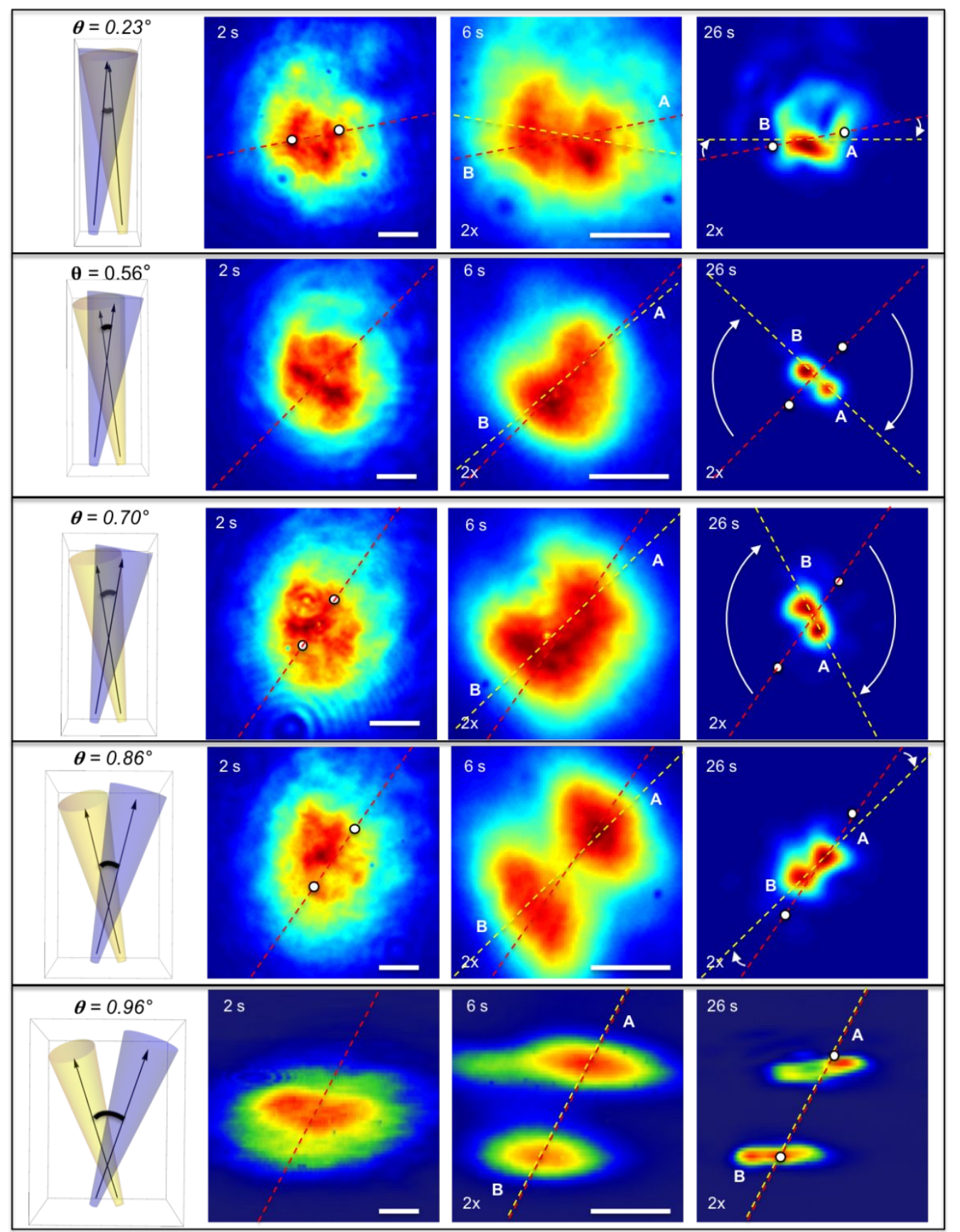

Figure S3 (a) Schemes of linear propagation (left) and polymerization-induced self-trapping and spiraling (right) of beams A (blue) and B (yellow). Experimental evolution of intensity profiles acquired at $z=6.0 \mathrm{~mm}$ at (top to bottom rows) $\theta=0.23^{\circ}, 0.56^{\circ}, 0.70^{\circ}, 0.86^{\circ}$ and $0.96^{\circ}$. White dots at $\mathrm{t}=2 \mathrm{~s}$ and $26 \mathrm{~s}$, represent beam-centres under linear conditions. Red and white dotted lines respectively show the initial and current orientation of beams. The scheme (left) depicts the beams' trajectories under linear conditions. Scale bar $=25 \mu \mathrm{m}$. For clarity, some images are 2x magnified. 


\section{References}

(S1) Villafranca, A. B.; Saravanamuttu, K. An Experimental Study of the Dynamics and Temporal Evolution of Self-Trapped Laser Beams in a Photopolymerizable Organosiloxane. J. Phys. Chem. C 2008, 112 (44), 17388-17396.

(S2) Morim, D. R.; Vargas-Baca, I.; Saravanamuttu, K. Reversibly Trapping Visible Laser Light through the Catalytic Photo-Oxidation of $\mathrm{I}^{-}$by $\mathrm{Ru}(\mathrm{bpy}){ }_{3}{ }^{2+}$. J. Phys. Chem. Lett. 2016, 7, $1585-1589$. 